\title{
Creación de aulas inclusivas, una propuesta para la atención de estudiantes con discapacidad intelectual
}

\author{
Creation of Inclusive Classrooms: A Proposal for Serving \\ Students with Intellectual Disabilities
}

Leila Yineth Gamboa Palacios*

\section{RESUMEN}

Ante la problemática sobre la atención a la población en condición de discapacidad intelectual, la presente propuesta pedagógica surge para dar respuesta a $i$ cómo fortalecer de manera pertinente el proceso de educación inclusiva de estudiantes con discapacidad intelectual de básica primaria, de la Institución Educativa Distrital John F. Kennedy, de acuerdo con lo establecido en el decreto 1421 del 29 de agosto de 2017? La investigación de enfoque cualitativo, de tipo investigación-acción, aplicó, para la recolección de la información, entrevistas semiestructuradas de preguntas abiertas a once docentes de primaria, tres directivos docentes y tres padres de familia de estudiantes con discapacidad intelectual, la cual se trianguló con componente referencial y legal para su análisis y resultado. Para un soporte teórico se tuvo presente a Lev S. Vygotsky, con su teoría sociocultural, y a Howard Gardner con la teoría de las inteligencias múltiples. La investigación concluye con el diseño de una propuesta pedagógica que consta de dos partes, la primera se ocupa de la legislación sobre educación inclusiva y, la segunda, de acciones pedagógicas concretas en el aula que mejorarán los procesos de enseñanza en los docentes y de aprendizaje en los estudiantes, en el marco de la Educación Para Todos, basada en la incorporación del Aprendizaje Cooperativo, de Johnson \& Johnson, en el desarrollo de contenidos temáticos en las diferentes áreas.
Palabras clave:

inclusión, educación inclusiva, discapacidad intelectual, aprendizaje cooperativo.

* Especialista, Maestría en Educación - Fundación Universitaria Los Libertadores, Bogotá, Colombia. Institución Educativa Distrital John F. Kennedy, Bogotá, Colombia. Contacto: lygamboap@libertadores.edu.co 


\begin{abstract}
Given the problem of serving members of the population with intellectual disabilities, this pedagogical proposal arises in response to the question of how to pertinently strengthen the inclusive education process for elementary students with intellectual disabilities at the John F. Kennedy District Educational Institution, according to the provisions of decree 1421 of August 29, 2017. This action research study takes a qualitative approach to collect information from semi-structured interviews with open questions, applied to eleven elementary school teachers and three parents of students with intellectual disabilities, which was triangulated with a referential and legal component for its analysis and results. This study was based on the sociocultural theory of Lev S. Vygotsky and the theory of multiple intelligences by Howard Gardner. The research concludes with the design of a two-part pedagogical proposal, the first part referring to legislation on inclusive education and, the second, concrete pedagogical actions in the classroom to improve the teachers' teaching processes and the students' learning processes, within the framework of Education for All, based on the incorporation of Cooperative Learning by Johnson \& Johnson, in the development of thematic content in different areas.
\end{abstract}

Key words: inclusion, inclusive education, intellectual disability, cooperative learning. 


\section{Introducción}

La educación inclusiva tiene por objeto eliminar la exclusión social como consecuencia de actitudes y respuestas a la diversidad en términos de raza, clase social, origen étnico, religión, género y aptitudes. Parte de la convicción de que la educación es un derecho humano fundamental y el cimiento de una sociedad más justa. En este sentido, es una manera de asegurar que la Educación Para Todos llegue realmente a todos; es un proceso que conlleva la transformación de las escuelas y otros centros de aprendizaje para atender a todos los estudiantes, incluidos los que presentan algún tipo de discapacidad, tanto física como intelectual; se lleva a cabo en muchos contextos, formales y no formales, en las familias y en la comunidad en su conjunto (UNESCO, 2008).

El termino "inclusión" ha evolucionado en las últimas décadas, originando en la actualidad un enfoque diferente en las políticas públicas de educación inclusiva. En la década de los 80 se utilizó el término "integración", refiriéndose a un principio que orienta, ayuda y mentaliza a aceptar que la sociedad está formada por seres diferentes, concibiendo así la integración desde una perspectiva social y reformulando el contexto para responder a una integración no sólo física, sino a un principio de normalización en el que los alumnos sean aceptados y atendidos de acuerdo con sus características.

De esta forma, la normalización se convierte en una base para la integración, en especial para la integración escolar, "entendida como la unificación de las educaciones ordinaria y especial, ofreciendo una serie de servicios a todos los niños y niñas de acuerdo a sus necesidades de aprendizaje" (Gaviria, 1992, p. 10, citado por Valecia, 2012).

En la década de los 90 se tiende a abandonar el término "integración" y a sustituirlo por el de "inclusión", por ser una época en la que se comienza el reconocimiento de los derechos de las personas en situación de discapacidad. El concepto de "inclusión" comunica, más claramente y con mayor exactitud, que todos los niños y niñas necesitan estar incorporados a la vida educativa y social de las escuelas, del barrio y de la sociedad en general, no únicamente dentro de la escuela ordinaria.

Es así como en Colombia, por primera vez en nuestra historia, se reconoce la necesidad de que el Estado promueva y garantice condi- 
ciones de igualdad, protección y atención educativa a las personas con discapacidad. Los artículos 3, 13, 47, 54 y 68 de la Constitución Política de Colombia, de 1991, establecen disposiciones específicas de atención en salud, educación y empleo para este colectivo.

Con la Ley General de Educación, capítulo 1, artículos 46 y 49, se establece que la educación para personas con limitación debe formar parte del servicio público educativo. En el artículo 46 se solicita a los establecimientos educativos que coordinen las acciones pedagógicas que juzguen pertinentes para atender las necesidades educativas de las personas con discapacidad. (Ministerio de Educación Nacional, 2017).

Con el decreto 1421 del 29 de agosto de 2017, que tiene como objetivo fundamental reglamentar la prestación del servicio educativo para la población con discapacidad, desde el acceso, la permanencia y la calidad, para que los niños puedan transitar por la educación desde preescolar hasta la superior o educación para el trabajo y el desarrollo humano, se quiere hacer efectiva en Colombia la atención educativa a la población con discapacidad que se enmarca en los principios de la educación inclusiva: calidad, diversidad, pertinencia, participación, equidad e interculturalidad establecidas por la Ley 1618 de 2013.

Por ello, la Institución Educativa Distrital John F. Kennedy, en su intento de cumplir con lo establecido en el decreto 1421, abrió sus puertas a estudiantes en condición de discapacidad intelectual, incorporándolos al aula regular junto con más de 30 compañeros con características totalmente diferentes, sin tener en cuenta los requerimientos de dicho decreto.

Frente a esa situación, los maestros de aula expresaban sentirse solos en esta tarea, porque, aunque es de su competencia, no cuentan con las herramientas necesarias para enfrentar este nuevo reto. Cuando recibían en el salón a uno o varios estudiantes con discapacidad intelectual, no sabían qué hacer con ellos, no existía una preparación previa para atenderlos.

En razón a esta problemática, para el desarrollo de la presente investigación se planteó como objetivo general "fortalecer de manera pertinente el proceso de educación inclusiva de estudiantes con discapacidad intelectual en Básica Primaria de la Institución Educativa 
Distrital John F. Kennedy", de acuerdo con lo establecido en el decreto 1421, para lo cual se propuso desarrollar, en este artículo, dos objetivos específicos:

1. Conocer la percepción que tiene la comunidad educativa Johnefista frente al proceso de educación inclusiva actual.

2. Diseñar una propuesta pedagógica que fortalezca la inclusión educativa en el grado tercero del John f. Kennedy IED.

En este orden de ideas, se hace necesario soportar esta investigación en unos postulados teóricos. Es así como se refiere a Lev S. Vygotsky que, con su enfoque, asignó un papel fundamental y formativo a las prácticas socioculturales en el desarrollo humano. Podemos darnos cuenta de que la idea de central de Vygotsky radica en que los seres humanos aprendan en entornos socioculturales, de ahí que la escuela pueda considerarse un dispositivo cultural orientado a producir ciertos tipos de desarrollo, a generar zonas de desarrollo próximo con el apoyo y la guía de quienes más saben.

En tal sentido la "zona de desarrollo proximal", es un constructo entre lo que un niño puede hacer independientemente, representando su nivel real de desarrollo, y lo que el niño puede hacer con la ayuda de otros, representando el nivel proximal de desarrollo. Esto coloca al maestro en el centro del proceso de aprendizaje, junto con los niños en su clase (Ruiz Carrillo \& Estrevel Rivera, 2010)

En la Teoría de Vygotsky, es fundamental que la participación infantil en actividades culturales, bajo la guía de compañeros más capaces, permita al niño interiorizar los instrumentos necesarios para pensar y acercarse a la resolución del problema de un modo más maduro que el que pondría en práctica si actuara por sí solo.

De esta manera, Vygotsky, con su enfoque sociocultural, hace un aporte muy valioso a la creación de aulas inclusivas, al permitir que los estudiantes en condición de discapacidad intelectual y los que no lo son, interactúen entre sí con el fin de mejorar los procesos de enseñanza y de aprendizaje, al propiciar ambientes ideales para generar zonas de desarrollo próximo en los estudiantes

Por otro lado, Howard Gardner, plantea la teoría de las inteligencias múltiples como una estrategia didáctica para atender a la diversidad 
y aprovechar el potencial de todos los alumnos. No niega el componente genético, pero insiste en que las inteligencias se pueden activar o inhibir en función de las oportunidades que se ofrecen o se dejan de ofrecer a un individuo. Así, el autor identifica ocho tipos de inteligencia diferentes (lingüística, lógico-matemática, visual-espacial, musical, cinético-corporal, interpersonal, intrapersonal y naturalista), todas importantes y necesarias para la vida, y presentes en todas las personas, aunque combinadas de una manera única en cada individuo. Esta combinación de inteligencias es lo que él llama "perfil intelectual" y nos lleva a asumir que todos somos diferentes y que, por lo tanto, no todos aprendemos de la misma manera.

Cabe aclarar que cada una de las inteligencias tiene más relevancia en un campo determinado, aunque no se pueda prescindir de las que en un principio parecen menos relevantes. Por este motivo la escuela debe favorecer el desarrollo de todas ellas, ya que el hecho de que una persona desarrolle más o menos sus inteligencias depende tanto de la herencia genética como de las experiencias vividas (Gardner, 2001). Para dar respuesta a esta diversidad intelectual apuesta por una escuela centrada en el individuo - que tenga en cuenta las características individuales de los alumnos y se preocupe de que todos aprendan de manera significativa-, ofreciéndonos un modelo abierto y flexible que permite su adaptación a cualquier realidad educativa; de hecho, se aplica en numerosos centros educativos del mundo, consiguiendo resultados muy positivos (Nadal Vivas, 2015).

Para la Educación Inclusiva en el John F. Kennedy, la teoría de las inteligencias múltiples cobra gran relevancia, en el sentido de que se potencian las habilidades de cada estudiante; lo que exige gran motivación de los maestros para atender a la población con discapacidad intelectual y que salgan de la rigidez del estricto cumplimiento con el plan de estudios. Es preciso flexibilizar el curriculum para que el estudiante aprenda a su propio ritmo, sin el afán de llenar unos contenidos temáticos.

Asimismo, en esta investigación, "Creación de aulas inclusivas para estudiantes con discapacidad intelectual en básica primaria”, se hace necesario aclarar algunos conceptos claves que ayudaran a fortalecer la propuesta pedagógica del presente artículo. 


\section{Inclusión educativa}

El reconocimiento de los derechos de todas las personas dentro de la sociedad, se visualiza en su participación en todos los contextos, siendo el área educativa donde más relevancia alcanza este principio. Entonces, la práctica docente debe propender por estrategias que permitan a cada alumno recibir un proceso educativo con el cual se logre el aprendizaje real, basado en sus capacidades y habilidades. "La educación inclusiva no es una cuestión marginal, sino que es crucial para lograr una educación de calidad para todos los educandos y para el desarrollo de sociedades más inclusivas" (ONU, 2008, p. 5).

Macarulla (2009) nos define "inclusión educativa" como un proceso de transformación en el cual los centros educativos se desarrollan en respuesta a la diversidad del alumnado de la escuela, identificando y eliminando las barreras que el entorno les pone, imposibilitando su aprendizaje, socialización y participación, pero también implica no dejar de atender a los alumnos a partir de sus capacidades y potencialidades (Macarulla, 2009).

Ainscow define "inclusión educativa" como un proceso de mejora e innovación educativa sistemático, para promover en los centros escolares la presencia, el aprendizaje y la participación de alumnos y alumnas en la vida escolar, en la que son escolarizados, con particular atención, aquellos más vulnerables. Para avanzar en esta dirección y en coherencia con una perspectiva social de la desventaja, es imprescindible detectar, eliminar o minimizar las barreras de distinto tipo que limitan dicho proceso (Ainscow, 2003)

Según el decreto 1421, la educación inclusiva es un proceso permanente, que reconoce, valora y responde de manera pertinente a la diversidad de características, intereses, posibilidades y expectativas de niñas, niños, adolescentes, jóvenes y adultos, cuyo objetivo es promover su desarrollo, aprendizaje y participación, con pares de su misma edad, en un ambiente de aprendizaje común, sin discriminación o exclusión alguna, y que garantiza, en el marco de los derechos humanos, los apoyos y los ajustes razonables requeridos en su proceso educativo, a través de prácticas, políticas y culturas que eliminan las barreras existentes en el entorno educativo (Decreto 1421,2017 , p. 5). 
Todas las definiciones coinciden con que la educación inclusiva es un proceso de transformación, en el cual los centros educativos se desarrollan en respuesta a la diversidad, con el fin de eliminar las barreras de exclusión en el entorno educativo que han existido en la historia de la humanidad. Esto exige a los docentes replantar sus prácticas pedagógicas dentro del aula, y a las instituciones ajustar un currículo global que dé respuesta a todos y todas.

\section{Discapacidad intelectual}

Durante las últimas décadas hemos presenciado un fuerte debate sobre cómo denominar, conceptualizar y abordar la discapacidad intelectual; después de muchas discusiones, lideradas por Miguel Ángel Verdugo a finales del siglo XX, la Confederación Española de Organizaciones en favor de Personas con Discapacidad Intelectual aprueba el término "discapacidad intelectual", frente al de "retraso mental" que, a pesar de ser un concepto clínico y psicológico formal, usado durante varias décadas, fue eliminado por la Asociación Americana de Discapacidades Intelectuales y del Desarrollo (AAIDD), al considerarlo una expresión peyorativa que subraya lo negativo para referirse a las personas con limitaciones de aprendizaje.

De esta forma, la AAIDD define de manera oficial la discapacidad intelectual como "una discapacidad caracterizada por limitaciones significativas tanto en el funcionamiento intelectual como la conducta adaptativa tal como se ha manifestado en habilidades adaptativas conceptuales, sociales y prácticas. Esta discapacidad comienza antes de los 18 años" (AAIDD, 2011).

El desarrollo histórico de la comprensión de la discapacidad intelectual evidencia cómo se ha pasado de una visión individualista a una perspectiva socioecológica, expresada en la interacción de la persona y su entorno, y en la que los apoyos desempeñan un papel importante en la mejora del funcionamiento humano. Esta nueva forma de abordarla muestra un avance cada vez mayor en brindar trato adecuado y en igualdad de condiciones a estas personas. Esto se refleja en el uso de términos más integradores y menos estigmatizadores, en reemplazo de términos peyorativos como "idiota", "imbécil" o "retrasado", usados históricamente para denominar a esta población. 
Lograr una educación inclusiva en las instituciones educativas es una gran responsabilidad que recae directamente en los docentes y directivos docentes, por cuanto constituyen el eje central que direcciona el proceso educativo. Tienen entre sus propósitos liderar las acciones en cuanto a la concertación del currículo, el plan de estudios, los proyectos transversales y la evaluación. En lo que se refiere a la flexibilización curricular, éste debe centrarse más en la metodología, en la didáctica, en los contenidos procedimentales y actitudinales, antes que en los contenidos conceptuales. Capacidades o talentos excepcionales en la educación inicial, básica y media. MEN (Rendón Arredondo, 2017, p. 26).

\section{Aprendizaje cooperativo}

El aprendizaje cooperativo no es un recién llegado al mundo de la educación; estamos hablando de un tema que aparece en los sesenta y que ha sido objeto de multitud de investigaciones que demuestran su efectividad en relación con los logros académicos y el desarrollo afectivo, cognitivo y social del individuo (Trujillo Sáez y Ariza Pérez, 2006, p. 35).

El aprendizaje cooperativo es el empleo didáctico de grupos reducidos, en los cuales los alumnos trabajan juntos para maximizar su propio aprendizaje y el de los demás. Contrasta con el aprendizaje competitivo, en el que cada alumno trabaja en contra de los demás para alcanzar objetivos escolares, tales como una calificación máxima que solo uno o algunos pueden obtener, y con el aprendizaje individualista, en el que los estudiantes trabajan por su cuenta para lograr metas de aprendizaje desvinculadas de las de los demás alumnos. En tal sentido, el docente puede organizar cooperativamente tareas didácticas, de cualquier materia y dentro de cualquier programa.

Esta estrategia pedagógica permite trabajar en tres clases de grupos de aprendizajes. Los grupos formales de aprendizaje cooperativo funcionan durante un periodo que va de una hora a varias semanas de clase. Los grupos informales de aprendizaje cooperativo operan durante unos pocos minutos, hasta una hora de clase; al igual que los grupos formales de aprendizaje cooperativo, los grupos informales sirven al maestro para asegurarse de que los alumnos efectúen el trabajo intelectual de organizar, explicar, resumir e integrar el material a las estructuras conceptuales existentes durante las actividades de enseñanza directa. 
Los grupos de base cooperativos tienen un funcionamiento de largo plazo (por lo menos de casi un año) y son grupos heterogéneos de aprendizaje con miembros permanentes, cuyo principal objetivo es posibilitar que sus integrantes se brinden unos a otros el apoyo para tener un buen rendimiento escolar.

Los grupos de base permiten que los alumnos entablen relaciones responsables y duraderas que los motivarán a esforzarse en sus tareas, a progresar en el cumplimiento de sus obligaciones escolares (como asistir a clase, completar todas las tareas asignadas, aprender) y a tener un buen desarrollo cognitivo y social (Johnson, Johnson y Holubec, 1999).

Para organizar sus clases de modo de que los alumnos realmente trabajen en forma cooperativa, el docente debe saber cuáles son los elementos básicos que hacen posible la cooperación. El conocimiento de estos elementos le permitirá:

- Tomar sus clases, programas y cursos actuales, y organizarlos cooperativamente.

- Diseñar clases cooperativas que se ajusten a sus propias necesidades y circunstancias pedagógicas, a sus propios programas de estudios, materias y alumnos.

- Diagnosticar los problemas que puedan tener algunos alumnos para trabajar juntos, e intervenir para aumentar la eficacia de los grupos de aprendizaje (Ibíd.).

\section{Diseño Universal para el Aprendizaje (DUA) y el decreto 1.421}

El DUA ha sido desarrollado por el Centro de Tecnología Especial Aplicada, CAST. Surge para dar respuesta a la diversidad en tanto se desee garantizar la equidad educativa, es decir, asegurar que a cada estudiante se le proporcione aquello que necesita en su proceso de aprendizaje. La atención a la diversidad se constituye, por tanto, como una cuestión de justicia. Por tal razón centra su foco de atención en el diseño del currículo escolar para explicar por qué hay alumnos que no llegan a alcanzar los aprendizajes previstos.

El DUA hace grandes aportes a la educación inclusiva y a la atención a la diversidad, como el de romper la dicotomía entre alumnado con discapacidad y sin discapacidad. La diversidad es un concepto que 
se aplica a todos los estudiantes, pues cada uno posee capacidades diferentes que se desarrollan en mayor o menor grado: cada cual aprende mejor de una forma única y diferente al resto. Por tanto, ofrecer distintas alternativas para acceder al aprendizaje no solo beneficia al estudiante con discapacidad, sino también permite que cada alumno escoja aquella opción con la que va a aprender mejor.

Por otro lado, aboga por un currículo flexible en el que quepan todos los estudiantes, empleando materiales y los medios en particular -infografías, videos subtitulados, textos digitales, entre otros- al diseño curricular en general. El currículo será discapacitante en la medida en que no permita que todo el alumnado pueda acceder a él (Pastor y Sánchez Serrano, 2014).

En Colombia, el DUA se establece dentro del decreto 1.421 de 2017, y contempla un conjunto de estrategias de inclusión para potenciar las posibilidades de aprendizaje de los estudiantes, que tiene en cuenta la diversidad en el aula y que permite a los maestros una metodología flexible. Pretende un acercamiento del maestro a los estudiantes desde el uso de diversos medios de aprendizaje.

Entonces, es importante que en la Institución Educativa John F. Kennedy los docentes desacomoden las tradicionales prácticas de aula y centren la atención en el estudiante como eje del proceso, con una propuesta incluyente como es el Diseño Universal de Aprendizaje, una estrategia que permite el uso de recursos y herramientas pedagógicas para desarrollar las habilidades y competencias, teniendo en cuenta los estilos y ritmos de aprendizajes, y que además permite utilizar una misma metodología de trabajo para todos los estudiantes, haciendo el currículo flexible que tiene en cuenta las diferencias individuales, permitiendo la educación para todos.

\section{Método}

El enfoque sobre el cual se aborda este proceso de investigación es de corte cualitativo. Blasco Mira y Pérez Turpín (2017) señalan que "en la investigación cualitativa se estudia la realidad en su contexto natural y cómo sucede, sacando e interpretando fenómenos de acuerdo con las personas implicadas" (p. 17). Los investigadores cualitativos tratan de comprender a las personas dentro del marco de referencia de ellas mis- 
mas. Desde un punto de vista fenomenológico, para la investigación cualitativa es esencial experimentar la realidad tal como otros la perciben. Siendo de esta manera que el investigador cualitativo se identifica con las personas que estudia para poder comprender cómo ven las cosas.

Por tal razón, este enfoque cualitativo permite a esta autora desarrollar la presente investigación en la Institución Educativa John F. Kennedy donde labora, involucrando a docentes, directivos docentes y padres de familia, dentro de su ambiente laboral y como parte de la experiencia en la atención de estudiantes en condición de discapacidad intelectual, ya que en su aula de clase, curso 303, integrado por 35 estudiantes entre 8 y 12 años de edad, se encuentra una estudiante con dicha condición, caso de inspiración para realizar este estudio.

La presente investigación es de tipo investigación-acción, ya que deja a un lado la postura teórica que adopta para cambiar la situación hasta conseguir una comprensión más profunda del problema práctico, siendo éste el propósito de toda investigación-acción (Elliot, 2000).

De igual manera, está adscrita a la línea de Evaluación, Aprendizaje y Docencia de la Fundación Universitaria Los Libertadores.

La población con la cual se llevó a cabo la investigación está ubicada en el suroccidente de la ciudad de Bogotá, localidad octava, en la Institución Educativa Distrital John F. Kennedy, ubicada en el barrio que lleva su nombre; está formada por 17 profesores de primaria, tres directivos de la Institución Educativa, una profesional de apoyo a la inclusión y seis padres de familia de estudiantes con discapacidad intelectual, para un total de 27 personas, de las cuales sale una muestra de once profesores de primaria, tres directivos y tres padres de familia, para una muestra de 17 personas.

Para el desarrollo de esta propuesta, se han planteado dos objetivos, asimismo se presentan dos fases que responden a cada uno de ellos. La primera, denominada "Percepción de la comunidad", se propuso conocer la apreciación que tenían los maestros, padres de familia, directivos docentes del John F. Kennedy IED acerca del proceso de inclusión. Para este propósito se emplearon las entrevistas semiestructuradas de preguntas abiertas, acompañadas de notas de campo y la observación participante para la recolección de la información, instrumentos que fueron aplicados a la muestra seleccionada. 
Para el análisis de la información, se establecieron las siguientes categorías:

\begin{tabular}{|c|c|c|}
\hline Objetivos específicos & Categorías & Subcategorías \\
\hline \multirow{4}{*}{$\begin{array}{l}\text { Conocer la percepción } \\
\text { que tiene la comunidad } \\
\text { educativa Johnefista } \\
\text { frente al proceso de } \\
\text { educación inclusiva } \\
\text { actual. }\end{array}$} & Educación inclusiva & $\begin{array}{l}\text { Concepto } \\
\text { Protocolo de atención }\end{array}$ \\
\hline & $\begin{array}{l}\text { Discapacidad } \\
\text { intelectual }\end{array}$ & $\begin{array}{l}\text { Diagnóstico } \\
\text { Rol del docente }\end{array}$ \\
\hline & Legislación & Decreto 1.421 \\
\hline & Currículo & $\begin{array}{l}\text { Estrategias pedagógicas } \\
\text { Evaluación } \\
\text { apoyos }\end{array}$ \\
\hline $\begin{array}{l}\text { Diseñar una propuesta } \\
\text { pedagógica que } \\
\text { fortalezca la inclusión } \\
\text { educativa en el grado } \\
\text { tercero del John f. } \\
\text { Kennedy IED. }\end{array}$ & \multicolumn{2}{|c|}{$\begin{array}{l}\text { Con base a los resultados obtenidos en el objetivo } \\
\text { específico número uno, se diseñó una propuesta } \\
\text { "Creación de aulas inclusivas, una propuesta } \\
\text { para la atención de estudiantes con discapacidad } \\
\text { intelectual", con la cual se pretende dar respuesta } \\
\text { al objetivo general, fortalecer de manera } \\
\text { pertinente el proceso de educación inclusiva } \\
\text { de estudiantes con discapacidad intelectual } \\
\text { en Básica Primaria de la Institución Educativa } \\
\text { Distrital John F. Kennedy, de acuerdo con lo } \\
\text { establecido en el decreto } 1.421\end{array}$} \\
\hline
\end{tabular}

Fuente: elaboración propia, 2020.

El análisis de la información recolectada se realizó a través de la triangulación de las categorías con el contexto de los entrevistados y los referentes teóricos.

En la segunda fase, "diseño y socialización", se diseñó la propuesta pedagógica para fortalecer la educación inclusiva en el curso 303 del John F. Kennedy IED. Por cuanto, en este curso, se encuentra una estudiante en esta condición. Una vez diseñada la propuesta se socializó con la comunidad educativa: maestros, directivos y padres de familia de estudiantes con discapacidad intelectual. La idea es que también se aplique para toda la primaria y, por qué no, en toda la institución educativa.

\section{Propuesta pedagógica}

La presente propuesta pedagógica, consta de dos partes. La primera acerca de la cualificación de la comunidad educativa respecto del decreto 1.421 de 2017: "Por el cual se reglamenta en el marco de la educación 
inclusiva la atención educativa a la población con discapacidad". Este decreto contempla los lineamientos para atender la población con discapacidad en Colombia, recoge toda la normatividad en un documento; parte de la definición de educación inclusiva, ruta de atención, responsabilidad de las instituciones públicas y privadas, funciones de los docentes y de los padres de familia, así como la evaluación, y termina con la promoción de estudiantes. Por lo tanto, responde a la problemática existente que arrojó el análisis de la información a través de la triangulación realizada, cual es el desconocimiento parcial o total de la legislación sobre atención a la discapacidad, en especial, el decreto 1.421.

Para hacer efectiva esta estrategia, se desarrollarán las siguientes actividades:

1. Se elaborará una cartilla de 20 páginas, cada una de ellas con su respectiva ilustración, y agradables a la vista, fáciles de leer, que resume el decreto 1.421 "aterrizado" a la Institución Educativa John F. Kennedy, la cual será un insumo para el colegio que deberá convertirse en material de consulta permanente para los docentes, directivos docentes y padres de familia; en ella encontrarán respuestas rápidas a cualquier interrogante acerca de la educación inclusiva en las instituciones educativas.

2. Presentación en forma física de la cartilla y explicación del contenido. Se realizará una jornada pedagógica con todos los docentes de primaria, profesionales de apoyo a la inclusión, directivos docentes y padres de familia de estudiantes con discapacidad intelectual, en la sala de informática del colegio. Después de hacer la presentación en físico de la cartilla, se enviará al correo electrónico de cada participante. Los maestros y directivos, en forma organizada en el uso de la palabra, leerán en voz alta cada uno de los títulos de la cartilla y explicarán su contenido, a la vez que la autora va mostrando en el videobeam el mismo contenido. Después de la lectura de cada título, hacen comentarios y discusiones acerca del contenido.

3. Cada director de curso se encargará de hacer el proceso de internalización del decreto con sus estudiantes.

4. Terminada la jornada pedagógica, los participantes diligenciarán un formato de retroalimentación de la cartilla, sugerencias y recomendaciones. 
5. Por último, los maestros, directivos docentes y padres de familia responderán a entrevista semiestructurada para determinar los resultados de la propuesta pedagógica en función de si responde a la problemática detectada en la fase de análisis.

Socialización de la propuesta. Debido a la pandemia del COVID-19 por la que estamos pasando en el mundo entero, en Colombia el confinamiento empezó el 26 de marzo de 2020, pero en los colegios el 16 de marzo, razón por la cual no hubo más encuentros presenciales en las instituciones educativas del país, lo que impidió llevar a cabo de manera presencial las actividades planteadas en la propuesta. El propósito es hacerlo cuando pase esta emergencia sanitaria.

De todos modos, la socialización de esta parte de la propuesta se logró hacer por el celular, a través de la aplicación de WhatsApp, enviando la cartilla por este medio y recibiendo la retroalimentación de la misma forma.

La segunda parte de la propuesta pedagógica está dirigida a los docentes de las diferentes asignaturas, con el fin de mejorar sus prácticas pedagógicas dentro del aula; será beneficiada, además, toda la comunidad educativa, en especial los estudiantes que presentan condición de discapacidad intelectual, por lo que se les garantizará una educación de calidad, mediante la interacción con sus compañeros, en igualdad de condiciones y contando con aula inclusivas.

En tal sentido, se proponen acciones concretas para trabajar en el aula con todos los estudiantes, sin tener que retirar a los estudiantes con discapacidad intelectual del salón ni de sus compañeros. Esta estrategia pedagógica corresponde al aspecto práctico. Se propone la implementación de acciones concretas en el aula, para desarrollar con todos los estudiantes, con o sin discapacidad intelectual.

Como estrategia pedagógica se propone el aprendizaje cooperativo sustentado por los hermanos Johnson \& Johnson que, en las aulas inclusivas, toma fuerza por cuanto permite al docente alcanzar varias metas importantes al mismo tiempo: en primer lugar, ayuda a elevar el rendimiento de todos sus alumnos, incluidos tanto los especialmente dotados como los que tienen dificultades para aprender. En este documento se presentará un modelo de cómo implementar el aprendizaje cooperativo dentro del aula, en el grado tercero de básica primaria 
(Trujillo Sáez y Ariza Pérez, 2006) y con el desarrollo de una temática del área de ciencias sociales, pero puede ser aplicable a todas las asignaturas y en todos los grados de $0^{\circ}$ a $11^{\circ}$.

\section{Descripción del contexto}

Esta experiencia de aprendizaje cooperativo se realizará en el curso 303, del John F. Kennedy I.E.D, jornada mañana en el área de ciencias sociales. En el aula de clases van a participar los 35 estudiantes, 20 niños y 15 niñas. En el grupo de niñas se presenta una alumna con dificultades en su aprendizaje, diagnosticada con discapacidad intelectual leve; tiene 12 años, cursó tres veces primero y tres veces segundo, el tercer segundo lo realizó en este colegio, donde se le detectó su condición. En el colegio donde estudió los años anteriores, perdió el cupo por doble repitencia, pues ya había cursado dos veces segundo y tenía en antecedente de haber repetido tres veces primer grado. En el grupo de niños, tres de ellos son repitentes.

\section{Descripción del contenido temático}

El tema que se va a trabajar son las "Regiones naturales de Colombia" Para su desarrollo se ocuparán tres semanas, con una intensidad horaria de tres horas semanales, para un total de nueve horas de clase.

El material requerido para esta experiencia es el siguiente: material audiovisual (computador), fotocopias a color de toda la unidad, papel blanco, lápices, colores, marcadores, mapa grande de las regiones; cartulina, marcadores, material reciclable, como cáscaras de huevo, aserrín, granos de lentejas, entre otros.

\section{Los objetivos propuestos son los siguientes:}

1. Identificar las características de cada una de las seis regiones naturales de Colombia.

2. Conocer en qué región del país estamos ubicados.

3. Localizar geográficamente las regiones naturales de Colombia.

Los contenidos a desarrollar son los siguientes:

1. Región de la Amazonía. 2. Región Andina. 3. Región Caribe. 4. Región Insular. 5. Región de la Orinoquía. 6. Región Pacífica. 


\section{Conformación de grupos cooperativos}

Una de las ventajas de los aprendizajes cooperativos es que el docente puede conformar los grupos de manera estratégica. Se conformarán ahora dependiendo de las habilidades de cada estudiante, de tal forma que los que tienen facilidad de aprender y los que se demoran un poco más para asimilar contenidos no queden repartidos en diferentes equipos de trabajo. La idea es que se complementen mutuamente.

De esta manera toma vigencia la idea de central de Vygotsky, que radica en que los seres humanos aprendan en entornos socioculturales con el apoyo y la guía de quienes saben más. Conocida como la "zona de desarrollo próximo".

Del mismo modo, Gardner, con su teoría de las inteligencias múltiples, se basa en la idea de que no existe una única inteligencia, sino que ésta tiene múltiples facetas que deben ser cultivadas en las aulas. Así, todas las inteligencias son importantes y necesarias para la vida y están presentes en todas las personas, aunque combinadas de una manera única en cada individuo, es lo que él llama "perfil intelectual" y nos lleva a asumir que todos somos diferentes y que, por lo tanto, no todos aprendemos de la misma manera.

\section{Secuencia de actividades}

\section{Primera semana}

Se trabajará un bloque de dos horas: después de una pequeña charla introductoria al tema, se conformarán seis grupos cooperativos con seis integrantes; uno de ellos quedará con cinco; cada uno se llamará, grupo matriz $(\mathrm{Gm})$ y se le asignará una región: $\mathrm{Gm}$ 1. Región de la Amazonía; Gm 2. Región Andina; Gm 3. Región Caribe; Gm 4. Región de la Orinoquía; Gm 5. Región Pacífica; Gm 6. Región Insular. Cada integrante se volverá "experto" en su región". Los estudiantes se desplazarán a la sala de informática donde, de manera individual, cada uno en su computador, verá un video sobre todas las regiones de Colombia. Luego, cada estudiante verá el video sugerido sobre la región que le correspondió.

En la tercera hora, organizados en los grupos matrices, se entregará a cada uno de los integrantes una hoja de papel, lápiz, colores, marcadores. Dividir la hoja en seis espacios, en los cuales cada estudiante 
dibujará una característica de su región. Cada uno inicia a escribir en el primer espacio, luego van rotando la hoja a la derecha y cada uno va realizando su dibujo, hasta que todos terminen.

\section{Segunda semana}

En el bloque de dos horas se hará una reunión de expertos: en cada grupo se queda un integrante que esperará a los otros expertos de cada región, donde harán intercambio de conocimientos del tema trabajado en el grupo matriz; como resultado, harán una cartelera que contendrá los dibujos de las características de las regiones que hicieron en el grupo matriz, la cual pegarán en diferentes paredes del salón. Luego volverán a su grupo de origen y socializarán lo que compartieron en la reunión de expertos.

En la siguiente clase, de una hora, un representante de cada grupo, que será voluntario, pasará al frente y, con la ayuda del mapa sobre las regiones, hará la localización de las regiones y dirá una característica relevante de cada una, utilizando las carteleras que dejaron pegadas en la clase anterior. En la casa harán un pequeño resumen en el cuaderno, sobre cada una de las regiones naturales. La idea es involucrar a los padres de familia.

\section{Tercera semana}

Se vuelven a reunir los expertos para revisar el resumen de cada compañero, girando el cuaderno a la izquierda. La idea es que, en la revisión, cada experto complemente el resumen del compañero con el tema que le tocó en el grupo matriz.

En la siguiente clase, de una hora, en el grupo matriz se hará la autoevaluación de la unidad didáctica trabajada. Cada estudiante expresará cómo fue su participación en el grupo y cómo se sintió.

\section{Reflexión sobre la acción pedagógica}

El aprendizaje cooperativo permite a los estudiantes interactuar entre ellos, permitiendo que, con el aporte de uno y otro, se logre el aprendizaje. La educación inclusiva es una metodología efectiva, porque el estudiante que presenta la discapacidad puede aprender de manera espontánea, con la ayuda de sus compañeros; no tiene actividades diferentes ni se aísla del grupo; de esta manera, se crean ambientes de 
aprendizajes para todos y se trata de cerrar la brecha del estudiante que presenta discapacidad y el que no, como lo plantea el Diseño Universal del Aprendizaje (DUA).

\section{Resultados}

Para el primer objetivo específico "Conocer la percepción que tiene la comunidad educativa Johnefista frente al proceso de educación inclusiva actual", después del análisis de la información recolectada con la aplicación de una entrevista semiestructurada y la observación participante se obtienen los siguientes resultados:

Los directivos, cinco docentes y dos de los padres de familia entrevistados, tienen claro el concepto de "educación inclusiva"; los otros seis docentes creen que inclusión educativa es solamente integración de los estudiantes con discapacidad en el aula regular. El otro padre de familia, equivocadamente, asocia el concepto de educación inclusiva con "una persona especializada que debe prestar atención a sus hijos con discapacidad en el colegio".

El protocolo de atención a la población en condición de discapacidad es conocido por las directivas del colegio, mas no por la mayoría de los docentes y padres de familia. Los docentes, al remitir a los estudiantes con discapacidad a la profesional de apoyo a la inclusión, esperan tener apoyo por parte de ella, pero no es así. Por otro lado, los padres de familia creen que toda la responsabilidad de la atención de sus hijos con discapacidad es de los docentes de aula.

Cuando los estudiantes presentan un diagnóstico de discapacidad intelectual en su historia clínica, se sigue el protocolo de atención, aunque el maestro se sienta solo en esa tarea. La situación se vuelve más compleja cuando la observación de sucesos académicos y/o comportamentales indica que el estudiante presenta algún tipo discacidad y no está diagnosticado.

Los docentes muestran gran preocupación porque creen que, a pesar de agotar todos sus esfuerzos y llevar estrategias adecuadas para el trabajo de estudiantes en condición de discapacidad intelectual, no logran los objetivos propuestos. Consideran que la educación debe ser más personalizada y con un grupo de 35 estudiantes en el salón es difícil hacerlo; aseguran que no poseen el conocimiento para atenderlos, 
pues no son licenciados en educación especial, ni han recibido ningún tipo de capacitación para ello.

Tanto los directivos como los docentes y padres de familia presentan desconocimiento de las políticas públicas de inclusión. Aunque son conscientes de que existe el decreto 1.421, que regula la educación inclusiva en las instituciones educativas en el orden nacional, manifiestan que se encuentra solo "en el papel"; al interior de la institución hace falta socialización y apropiación de las políticas de inclusión.

Los docentes reconocen que carecen de herramientas pedagógicas para atender a la población en condición de discapacidad intelectual, debido a sus escasos conocimientos sobre el tema y, sumado a ello, no cuentan con el apoyo de la persona competente ni de los padres de familia; aun así, manifiestan gran interés por recibir capacitación para atender a esta población. Ven indispensable trabajar de la mano con la profesional de apoyo a la inclusión y con los padres de familia.

En cuanto a la evaluación, el panorama no cambia, se percibe un vacío en el tema porque se cree que los estudiantes deben ser promovidos sin tener en cuanta unos mínimos de exigencia en su desempeño académico. Así queda evidenciada la falta de un trabajo coordinado entre los diferentes estamentos del colegio, como las directivas, docentes de aula, docentes de apoyo pedagógico y padres de familia, para que se lleve a cabo de la mejor manera la atención a la población con discapacidad intelectual.

Con base en los resultados del objetivo número uno, se dio respuesta al objetivo número dos con el diseño de una propuesta pedagógica para fortalecer la educación inclusiva en el grado tercero del John f. Kennedy IED.

Al socializarla, presenta los siguientes resultados.

Le dan mérito al hecho de tener el decreto sintetizado de acuerdo con las necesidades del colegio, en una cartilla de fácil lectura y comprensión, que despeja las dudas sobre conceptos, proceso de inclusión en el aula, ruta de atención a la población con discacidad, rol del docente, de los profesionales de apoyo, directivos y padres de familia.

Afirman los docentes que muchas veces existe la información, pero por múltiples razones no se accede a ella. La propuesta que se 
hizo en la presente investigación, hace más expedita la consulta del decreto 1.421.

Con la propuesta pedagógica se resuelve la problemática que se detectó, cual fue el desconocimiento de una política de educación inclusiva, en especial del decreto socializado, la ruta y protocolo de atención a la población con discapacidad, hasta el concepto mismo de "educación inclusiva", como los roles de los docentes de aula, docentes de apoyo pedagógico y padres de familia dentro del proceso de atención a los estudiantes con discapacidad.

Al leer detenidamente la cartilla que sintetiza muy bien el decreto y lo aterriza al contexto de la institución educativa John F. Kennedy, los docentes reconocen que la responsabilidad de la atención a estudiantes con discapacidad intelectual recae sobre el docente de aula que, equivocadamente, pensaba que esto le correspondía más la profesional de apoyo a la inclusión. Pero realmente ella es una la guía, una orientadora de ese proceso. Teniendo claro todo esto es más fácil hacer el trabajo.

Los docentes y directivos que no respondieron frente a la socialización de la propuesta, expresaron no hacerlo por falta de tiempo, y que tan pronto lean el material presentarán su opinión. Ante esta situación se puede analizar que hay docentes que poco les gusta leer. Que por más que tengamos a la mano los documentos o leyes, no hay interés para hacerlo. En el caso de los tres docentes que no respondieron, ninguno de ellos tiene en el aula estudiantes con discapacidad, por lo tanto, se puede deducir la falta de interés por el tema. El otro padre de familia que no respondió, al parecer no le llegó la información.

En cuanto a la segunda parte de la propuesta pedagógica, dado que se propusieron acciones pedagógicas que se desarrollarán concretamente en las instalaciones del colegio, no se alcanzó a llevar a la práctica debido al estado de confinamiento al que están sometidas las personas del país por la pandemia del coronavirus. Por lo tanto, los resultados quedan pendientes para próximas investigaciones.

\section{Discusión y conclusiones}

Vale destacar que Colombia requiere profesionales en educación inclusiva. Profesores capacitados que planeen, gestionen, evalúen y ha- 
gan seguimiento a los procesos educativos de poblaciones diversas, para acogerlas en los diferentes niveles de la educación y contextos socioculturales, y que trabajen en la explotación de las capacidades humanas hacia el mejor desarrollo del individuo y los diferentes núcleos sociales en los que participa. Formar estos docentes se constituye en una prioridad (Arias Castilla \& Ayala Cardona, 2018).

Con el desarrollo de esta investigación, se pudo interactuar con algunos miembros de la comunidad educativa - directivos docentes, docentes de aula, docentes de apoyo y algunos padres de familia- de la Institución Educativa Distrital, John F. Kennedy, lo que permitió conocer la problemática que presenta dicha institución educativa en el proceso de educación inclusiva, como es el desconocimiento parcial o total del decreto 1.421, en el entendido que en este decreto se reglamenta la ruta, el esquema y las condiciones para la atención educativa a la población con discapacidad en los niveles de preescolar, básica y media. Se encuentra consignado las definiciones de educación inclusiva, currículo flexible, Diseño Universal del Aprendizaje DUA, entre otras, para mejorar la atención a esta población.

Para ello se recomienda, en primer lugar, la cualificación de los docentes en este campo por parte del Ministerio de Educación; los docentes de aula, que son los que atienden a la población con discapacidad, dicen carecer de la formación académica en esta área. Si por ley los maestros deben ser inclusivos, el Estado debe proporcionarles las herramientas necesarias para dar una educación de calidad a estos estudiantes; así, la formación del docente cobra atención como lo contempla el decreto 1.421, (p. 17).

En virtud de los resultados, se evidenció la falta de armonía y trabajo articulado de los docentes de aula con la profesional de apoyo a la inclusión, situación que repercute en la atención a estudiantes con discapacidad, pues se desconoce cuál es el papel que desempeña cada uno en este proceso. Por tal razón, se recomienda que cada estamento revise sus funciones y trabaje en relación con estas; pero, independientemente de ello, debe haber mayor comunicación y trabajo integrado, pensando siempre en los estudiantes y en mejorar el ambiente laboral.

En la Institución Educativa John F. Kennedy hay la necesidad de que las directivas, junto con la profesional de apoyo, den a conocer 
muy bien las políticas públicas de educación inclusiva a la comunidad por medio de diferentes estrategias, como lecturas, talleres, videos. No se debe quedar solamente en el papel: se debe interpretar, interiorizar y acordar dinámicas para llevarlas a la práctica, en respuesta a la formación permanente del profesorado. De ella dependen la calidad de la enseñanza y la capacidad para responder con eficacia y profesionalidad.

Para mejorar las prácticas pedagógicas se recomiendan acciones desde el Diseño Universal de Aprendizaje (DUA), que da cabida a todos los estudiantes desde un diseño de currículo flexible, en el que se tienen en cuenta las capacidades y realidades de cada estudiante. Además, ofrece diferentes estrategias que no excluyen, sino que incorporan las diferencias individuales, logrando la educación para todos. Desde el DUA se inicia un camino que cierra la brecha entre el estudiante con discapacidad y el estudiante sin discapacidad.

Así las cosas, se recomienda introducir en el aula de clases la metodología de aprendizaje cooperativo, como alternativa de enseñanza a los modelos tradicionales individualistas y competitivos, con el fin de mejorar el rendimiento académico, la autoestima y las habilidades sociales, además de incrementar la motivación y el gusto por aprender: los estudiantes aprenden en pequeños grupos estratégicamente conformados.

Mientras los docentes no reciban capacitación por parte del Estado, se recomienda que hagan uso del autoaprendizaje, que consulten por sus propios medios sobre estrategias pedagógicas para aplicar en el aula, pues lograr una educación inclusiva en las instituciones educativas, es una gran responsabilidad de los docentes y directivos docentes, por cuanto ellos constituyen el eje central que direcciona el proceso educativo. Tienen entre sus propósitos liderar las acciones en cuanto a la concertación del currículo, el plan de estudios, los proyectos transversales y la evaluación, sin dejar de lado la adaptación de escenarios de aprendizajes que vinculen al estudiante con los procesos para lograr el desarrollo de las competencias necesarias, a partir de los avances de la temática propuesta.

El diseño de los Planes Individuales de Ajustes Razonables, PIAR, es decir, ajustes curriculares, didácticos, evaluativos y metodológicos, entre otros, para el año lectivo, de los estudiantes que lo requieren, lo 
liderarán los docentes de aula con el docente de apoyo, la familia y el estudiante. Según la organización escolar, participarán los directivos docentes y el orientador decreto 1.421 (p. 12 y 13).

\section{Referencias}

AAIDD. (2011). Discapacidad Intelectual, Definición, Clasificacion y Sistemas de Apoyo Social. Madrid: Alianza Editorial.

Ainscow, M. (2003). Desarrollo de Sistemas Educativos Inclusivos. Recuperado de https://bit.ly/2WUAfpB.

Arias Castilla, C. y Ayala Cardona, J. (2018). Diversidad en Educación Infantil. Programas de formadores par la infancia en Colombia. Bogotá: Corporación Universitaria Iberoamericana.

Blasco Mira, J. E. y Pérez Turpín, J. A. (2017). Metodologías de Investigación en la Enseñanza de la Educación Física y el Deporte. Recuperado de: https://bit.ly/2VlLIxf.

Elliot, J. (2000). La investigación-acción en educación. Madrid: Morata, S. L.

Johnson, D., Johnson, R. y Holubec , E. (1999). El aprendizaje cooperativo. Buenos Aires: Editorial Paidós.

Macarulla, I. (2009). Buenas Prácticas de Escuela Inclusiva: La Inclusión de Alumnado con Discapacidad: Un reto, una necesidad. Barcelona: Graó.

MEN. (2017). Decreto 1421. Bogotá: Recuperado de: https://bit. ly/2xSQN7L.

Ministerio de Educación Nacional. (2017). Documento de orientaciones técnicas, administrativas y pedagógicas. Bogotá.

Nadal Vivas, B. (2015). Las inteligencias múltiples como una estrategia didáctica para atender a la diversidad y aprovechar el potencial de todos los alumnos. Revista de educación inclusiva, 8(3), 121-136.

Pastor, C. A. y Sánchez Serrano, J. M. (2014). Diseño Universal para el Aprendizaje (DUA) Pautas para su introducción en el currículo. Recuperado de: https://bit.ly/3dv9zk9.

Rendón Arredondo, M. (2017). Orientaciones para la transición educativa de los estudiantes con discapacidad y con capacidades o talentos excepcionales en la educación inicial, básica y media. Bogotá: Ministerio de Educación Nacional. 
Rogoff, B. (1993). Aprendices del pensamiento el desarrollo cognitivo en el contexto social. Barcelona: Paidós Ibérica.

Ruiz Carrillo, E. \& Estrevel Rivera, L. (2010). Vigotsky: la escuela y la subjetividad. Pensamiento Psicológico, 8(15), 135-145

Trujillo Sáez, F. y Ariza Pérez, M. Á. (2006). Experiencias Educativas en Aprendizaje Cooperativo. Grupo Editorial Universitario. Recuperado de: https://bit.ly/2CK1KdH.

UNESCO. (2008). La Educación Inclusiva: El Camino Hacia el Futuro. Centro Internacional de Conferencias (pág. 6). Ginebra: ED/BIE/CONFINTED 48/3. Recuperado de: https://bit. ly/2WN7FoF.

Valecia, A. (2012). Historia de la Inclusión Educativa. Colombia: Recuperado de: http://eduincluye.blogspot.com/.

Verdugo, M. Á. (2003). Análisis de la definición de discapacidad intelectual de la Asociación Americana sobre Retraso Mental. Recuperado de https://bit.ly/2AbLtgn. 